\title{
APLICAÇÃO DE MECÂNICA MOLECULAR EM QUÍMICA INORGÂNICA
}

Lilian Weitzel Coelho, Georgia Maria Amaral Junqueira, Juan Omar Machuca Herrera e Sérgio de Paula Machado. Departamento de Química Inorgânica - Instituto de Química da Universidade Federal do Rio de Janeiro - CT-Bloco A - Sala 632 - Ilha do Fundão, Cidade Universitária - 21945-970 - Rio de Janeiro - RJ

Bianca da Cunha Machado

Departamento de Química Geral e Inorgânica - Instituto de Química da Universidade Federal Fluminense - Outeiro de São João Batista s/nº Valonguinho - 24020-150 - Niterói - RJ

Recebido em 3/10/97; aceito em 2/12/98

\begin{abstract}
MOLECULAR MECHANICS APPLICATION IN INORGANIC CHEMISTRY. The present paper is a review about basic principles of the molecular mechanics that is the most important tool used in molecular modeling area, and their applications to the calculation of the relative stability and chemical reactivity of organometalic and coordination compounds. We show how molecular mechanics can be successfully applied to a wide variety of inorganic systems.
\end{abstract}

Keywords: molecular mechanics; coordination compounds; trans effect.

\section{INTRODUÇÃO}

$\mathrm{O}$ interesse em modelagem molecular utilizando recursos computacionais vem aumentando em função do melhor desempenho apresentado pelos computadores nas últimas duas décadas.

A modelagem molecular atualmente está sendo amplamente aplicada na interpretação de resultados experimentais e na construção de novos materiais com propriedades específicas.

A base da modelagem molecular está em relacionar todas as propriedades moleculares importantes, ou seja, estabilidade, reatividade e propriedades eletrônicas com a estrutura molecular. Desta forma é possível desenvolver algorítmos capazes de calcular uma estrutura molecular com uma estereoquímica específica e correlacioná-la com as propriedades moleculares apresentadas pela substância.

Existem três principais categorias de cálculos teóricos: método de orbital molecular ab initio, método de orbital molecular semi-empírico e cálculos de campo de força empírico também conhecido como mecânica molecular.

A mecânica molecular é um dos métodos que, principalmente por razões de simplicidade computacional e eficiência, tem sido o mais utilizado para modelar estruturas moleculares e energias conformacionais na elucidação de problemas experimentais em química e bioquímica.

Historicamente, os cálculos de mecânica molecular tiveram início em compostos orgânicos. Os primeiros cálculos foram realizados por Bartell et alli ${ }^{1}$ e Boyd et alli ${ }^{2}$. Allinger analisando as deficiências do que denominou "primeira geração" dos campos de força, onde estão incluídos os campos $\mathrm{MM}^{3}, \mathrm{EAS}^{4}$, Mub- $2^{5}$, desenvolveu o campo de força MM26 para hidrocarbonetos, no qual incluiu os termos de torção e o aprimorou gerando os campos de força $\mathrm{MM} 3^{7}$ para compostos orgânicos oxigenados. Posteriormente foram desenvolvidos campos de força para compostos inorgânicos ${ }^{8-10}$. Desde então mecânica molecular vem sendo utilizada no estudo de diferentes sistemas envolvendo íons metálicos ${ }^{11-16}$.

Este artigo tem como objetivo divulgar a aplicabilidade e eficiência da mecânica molecular na modelagem molecular de compostos inorgânicos, apresentando os conceitos básicos da mecânica molecular e alguns exemplos de suas aplicações em química inorgânica.

\section{CONCEITOS FUNDAMENTAIS DA MECÂNICA MOLECULAR}

A mecânica molecular é um importante representante dos métodos clássicos teóricos. A metodologia da mecânica molecular está baseada na aproximação de Born-Oppenheimer. Dentro desta aproximação os movimentos dos núcleos e dos elétrons podem ser tratados separadamente. Neste contexto a mecânica molecular é caracterizada por considerar os movimentos nucleares e por tratar os elétrons indiretamente, diferindo dos métodos quânticos de orbitais moleculares, caracterizados por estudar a estrutura eletrônica, para uma posição nuclear fixa. Na mecânica molecular é suposto que a densidade eletrônica pode ajustar-se instantaneamente a qualquer alteração na configuração geométrica dos núcleos, assumindo que os núcleos possuem movimento livre e que todas as interações nucleares são aditivas. A superfície de Born-Oppenheimer, conhecida na mecânica molecular como superfície de energia potencial, é multidimensional e descreve a energia de uma molécula em termos das posições nucleares. Esta superfície possui mínimos locais em relação a uma ou algumas conformações moleculares estáveis e, em uma primeira aproximação, a molécula é descrita pela estrutura correspondente ao mínimo global da superfície potencial.

Cálculos de mecânica molecular utilizam uma série de equações derivadas empiricamente para a superfície de BornOppenheimer, cujo formalismo matemático está baseado num modelo de mecânica clássica de estrutura molecular.

$\mathrm{O}$ modelo da mecânica molecular considera as moléculas como uma coleção de átomos ligados entre si por forças elásticas ou harmônicas. Estas forças são descritas pelas funções de energia potencial das contribuições estruturais, e.g. comprimentos de ligação (r), ângulos de ligação $(\theta)$, ângulos diedros $(\phi)$ e interações não ligadas (d). O conjunto destas funções é conhecido como campo de força empírico. O campo de força representa a energia potencial molecular em relação a uma geometria de referência, em um determinado sistema de coordenadas:

$$
U=\Sigma U(r)+\Sigma U(\theta)+\Sigma U(\phi)+\Sigma U(d)+\ldots
$$

A energia, U, de uma molécula, dentro do campo de força, é a soma de todas as contribuições de energia, onde U é, algumas 
vezes, conhecido como energia estérica da molécula e é considerada como a diferença entre a energia da molécula real e a energia da molécula hipotética. Nesta última todos os parâmetros estruturais são exatamente os valores ideais ou "naturais", definidos para uma situação hipotética onde não existem quaisquer tensões entre as coordenadas internas do sistema molecular. $U_{\mathrm{r}}$ ação a seu valor ideal, $\mathrm{U}_{\theta}$ é a energia de deformação do ângulo de ligação em relação a seu valor ideal, $U_{\phi}$ é a energia devido a torção em torno de uma ligação e $\mathrm{U}_{\mathrm{d}}$ é a energia da interação entre átomos não ligados. Outras interações intramoleculares que afetam a energia, como as interações eletrostáticas ou ligações hidrogênio, também podem ser adicionadas ao campo de força, assim como as interações cruzadas, representadas pelos chamados termos cruzados, as quais consideram um certo número de interações entre comprimentos de ligação, ângulos de ligação, ângulos diedros, interações carga-carga e interações dipolo-dipolo.

Não existem regras rígidas acerca do número ou tipos de funções de energia potencial a serem empregados. Esta escolha é feita de acordo com as propriedades moleculares selecionadas à reproduzir. Dependendo da função de energia potencial são permitidas as seguintes opções:

a) cálculos das propriedades geométricas;

b) cálculos das propriedades geométricas e vibracionais;

c) cálculos das propriedades geométricas e termodinâmicas;

d) cálculos das propriedades geométricas, vibracionais e termodinâmicas.

As expressões da energia potencial desenvolvidas para cálculos de mecânica molecular são todas descendentes de três tipos básicos de campo de força:

Campo de Força Central - considera apenas distâncias interatômicas (inclusive comprimentos de ligação).

Campo de Força de Valência Geral - considera comprimentos e ângulos de ligação, ângulos diedros, coordenadas de deformação fora do plano e adicionam os termos cruzados.

Campo de Força de Urey-Bradley - considera comprimentos e ângulos de ligação, ângulos diedros, coordenadas de deformação fora do plano e as interações entre átomos ligados por um centro comum.

Enquanto propriedades como geometrias moleculares de energias mínimas, alturas de barreiras de rotação interna, entalpias de formação e funções termodinâmicas são bem reproduzidas por um campo de força de valência simples (campo de força central, incluindo os ângulos de ligação, ângulos diedros e coordenadas de deformação fora do plano), o mesmo não acontece com as propriedades vibracionais, principalmente números de onda, os quais normalmente para serem bem reproduzidos necessitam da inclusão das interações entre átomos ligados por um centro comum (interações não ligadas 1,3), interações entre átomos não ligados (interações não ligadas 1,4 e superiores) e/ou das interações cruzadas.

A generalização de uma função potencial permite resultados com melhor concordância entre os resultados calculados e os experimentais. No entanto, um considerável trabalho matemático é requerido devido a complexidade assumida pela função potencial. A maior dificuldade está em selecionar quais dos termos cruzados devem ser considerados, uma vez que, é impossível incluir todas as interações cruzadas presentes em um sistema molecular. A escolha desses termos é empírica.

As funções de energia potencial mais utilizadas em cálculos de mecânica molecular, bem como a descrição da utilização dessa metodologia de cálculo, seus fundamentos filosóficos e aspectos matemáticos têm sido descritos em diversos trabalhos ${ }^{1-25}$.

\section{A Função de Energia Potencial para Cálculos de Mecânica Molecular}

A energia potencial, $U$, de uma molécula com $\mathrm{N}$ átomos, definida em termos das $3 \mathrm{~N}$ coordenadas, $x_{i}$, quando deformadas de sua geometria de energia potencial, $U_{0}$, pode ser escrita como uma expansão em séries de Taylor:

$U=U_{0}+\sum_{i=1}^{3 N}\left(\frac{\partial U}{\partial x_{i}}\right)_{0} \Delta x_{i}+\frac{1}{2} \sum_{i, j=1}^{3 N}\left(\frac{\partial^{2} U}{\partial x_{i} \partial x_{j}}\right)_{0} \Delta_{x_{i}} \Delta+$

$$
\sum_{i, j, k=1}^{3 N}\left(\frac{\partial^{3} U}{\partial x_{i} \partial x_{j} \partial x_{k}}\right)_{0} \Delta x_{i} \Delta x_{j} \Delta x_{k}+\text { termos superiores }
$$

O primeiro termo, $U_{0}$, é a energia total da molécula hipotética. As primeiras derivadas formam as $3 \mathrm{~N}$ componentes do vetor gradiente da energia potencial. As segundas derivadas são os elementos da matriz hessiana de dimensões $3 \mathrm{~N}$ por $3 \mathrm{~N}$, conhecida também como matriz das constantes de forças.

Considerando que os deslocamentos $\Delta \mathrm{x}_{\mathrm{i}}, \Delta \mathrm{x}_{\mathrm{j}}, \Delta \mathrm{x}_{\mathrm{k}}$ são infinitesimais, em torno do ponto de equilíbrio, os termos superiores aos quadráticos podem ser desconsiderados (aproximação harmônica) ${ }^{21}$.

Baseado na análise vibracional de uma molécula com geometria molecular correspondente ao mínimo de energia (geometria de equilíbrio), o termo $U_{0}$ pode ser assumido igual a zero e aplicando a definição de mínimo de energia potencial nesta geometria, o termo $\frac{\partial U}{\partial x_{i}}$, deve ser, teoricamente, igual a zero. Desta forma a energia potencial molecular depende somente do termo correspondente as segundas derivadas:

$U=\frac{1}{2} \sum_{i, j=1}^{3 N}\left(\frac{\partial^{2} U}{\partial x_{i} \partial x_{j}}\right)_{0} \Delta x_{i} \Delta x_{j}$

Substituindo na equação 3, as segundas derivadas por seus símbolos $\mathrm{f}_{i j}$, a relação de um campo de força harmônico é obtida:

$U=\frac{1}{2} \sum_{i, j=1}^{3 N} f_{i j} \Delta x_{i} \Delta x_{j}$

Esta equação descreve um sistema de osciladores harmônicos acoplados, onde os subscritos $i=j$ são os termos da diagonal principal e $i \neq j$ são os termos fora da diagonal da matriz das constantes de força. Quando apenas os termos diagonais são considerados os osciladores estão desacoplados:

$U=\frac{1}{2} \sum_{i=1}^{3 N} f_{i j} \Delta x_{i}^{2}$

A curva de energia potencial pode então ser descrita pela Lei de Hooke:

$U=\frac{1}{2} k(\Delta x)^{2}$

onde $\mathrm{k}$ é a constante de força do oscilador harmônico. Este resultado pode ser formulado em termos das coordenadas internas moleculares dando origem aos campos de força com potenciais harmônicos.

Para os deslocamentos, $\Delta x_{i}$, fora do limite de validade da aproximação harmônica, os termos superiores da equação 2 devem ser considerados. Isto significa que efeitos anarmônicos são incluídos. A aproximação mais utilizada para descrever os efeitos anarmônicos, consiste em truncar a expansão em séries de Taylor após o termo cúbico, para os comprimentos de ligação e para os ângulos de ligação. A função potencial para estas coordenadas assume a forma: 


$$
\begin{aligned}
& U=\frac{1}{2} k_{r}\left(r-r_{0}\right)^{2}+k_{r}^{\odot}\left(r-r_{0}\right)^{3} \\
& U_{\theta}=\frac{1}{2} k_{\theta}\left(\theta-\theta_{0}\right)^{2}+k_{\theta}^{\odot}\left(\theta-\theta_{0}\right)^{3}
\end{aligned}
$$

onde $K_{r}, K_{r}^{\prime}, K_{0}^{\prime}$, são os chamados parâmetros de energia e representam as constantes de força harmônicas (os índices subscritos denotam as coordenadas internas a que se referem). Este tipo de função é bastante adequado para comprimentos de ligação que não estejam muito afastados do limite demarcado pela lei de Hooke (Figura 1).

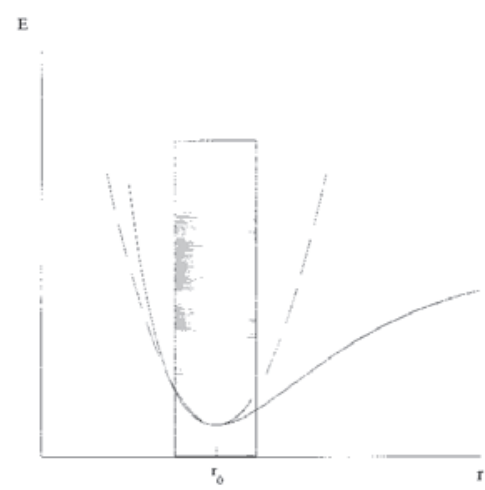

Figura 1. Curva de energia potencial para estiramento de uma ligação química. Curva sólida, função de Morse; curva tracejada, lei de Hooke.

Caso os comprimentos de ligação sejam suficientemente longos, de modo que a função cúbica não descreva a curva de energia potencial corretamente (deslocamentos $(\Delta \mathrm{x})$ grandes invertem a função cúbica), existe a possibilidade de substituir o potencial harmônico por um outro tipo de função de energia potencial como a função de Morse (figura 1):

$$
U=D_{0}\left[1-e^{-\alpha\left(r-r_{0}\right)}\right]^{2}-D_{0}
$$

onde $\mathrm{D}_{0}$ é a energia potencial de interação entre os átomos quando $\mathrm{r}=\mathrm{r}_{0}$ e $\mathrm{a}$, um parâmetro adicional que pode ser relacionado com a frequiência vibracional, $v$, de uma molécula diatômica:

$$
v=\frac{1}{2 \pi} \sqrt{\frac{k}{\mu}}
$$

onde $\mu$ é a massa reduzida para a vibração e $k$ a constante de força, equivalente a segunda derivada da função potencial:

$$
k=\frac{d^{2} U}{d r^{2}}
$$

então

$$
\mathrm{k}=2 \mathrm{D}_{0} \alpha^{2}
$$

$\log 0$

$$
\alpha=\sqrt{\frac{k}{2 D_{0}}}
$$

A utilização da função de Morse não é muito comum em mecânica molecular. A sua maior complexidade requer um tempo de computação superior quando comparado a lei de Hooke, de modo que a maioria dos campos de força disponíveis não incluem funções do tipo Morse, optando por outros tipos de refinamentos, principalmente na função torsional, onde a função de energia potencial:

$U_{\phi}=\frac{1}{2} K_{\phi}\left(\phi-\phi_{0}\right)^{2}$

é substituída por uma função periódica do tipo:

$U_{\phi}=\frac{1}{2} K_{\phi}(1+s \cos n \phi)$

onde $\mathrm{s}=+1$ ou -1 , dependendo se existe ou não conformação eclipsada $(\phi=0)$ em algum ponto da estrutura molecular $(\phi=0$ corresponde ao máximo ou mínimo da energia torsional) e $n$ é a periodicidade da energia potencial torsional, ou por uma expansão em séries de Taylor, normalmente truncada após o terceiro termo:

$U_{\phi}=\Sigma\left[\frac{1}{2} V_{1}(1+\cos \phi)+\frac{1}{2} V_{2}(1+\cos 2 \phi)+\frac{1}{2} V_{3}(1+\cos 3 \phi)+\ldots\right]$

onde os parâmetros, $\mathrm{V}_{1}, \mathrm{~V}_{2}$ e $\mathrm{V}_{3}$, são ajustados de modo que as conformações previstas pela teoria reproduzam os resultados experimentais para algumas moléculas testes.

Outro refinamento importante e bastante utilizado, consiste na inclusão de um termo adicional, $\mathrm{U}_{\mathrm{VdW}}\left(\Sigma \mathrm{U}_{\mathrm{VdW}}\right)$ na equação 1 , para descrever em função da distância entre os núcleos as interações não ligadas entre átomos separados por mais do que duas ligações. A energia potencial total das interações entre um par de átomos é a soma das energias das forças atrativas (forças de dispersão de London) e das forças repulsivas (repulsões de Van der Waals). As duas funções de energia potencial mais utilizadas para descrever estas interações são o potencial de LenardJones (ULJ) e o potencial de Buckingham $\left(\mathrm{UB}_{\mathrm{uck}}\right)$ :

$\mathrm{U}_{\mathrm{LJ}}=\frac{\mathrm{A}}{\mathrm{r}^{12}}-\frac{\mathrm{B}}{\mathrm{r}^{6}}$

$U_{B u c k}=A @ \exp (B \Phi r)-C / r^{6}$

onde r é a distância entre dois núcleos e A, B, C são parâmetros baseados em constantes atômicas dependentes dos átomos envolvidos.

Alguns autores complementam as expressões 17 e 18 com termos do tipo $\mathbf{q i q}_{\mathbf{j}} / \mathrm{r}_{\mathrm{ij}}$ (q: carga parcial eletrônica) ${ }^{26}$.

O objetivo principal, na construção de um campo de força, está em poder transferí-lo de uma molécula para outra. Os parâmetros são selecionados para reproduzir os dados experimentais para alguns compostos testes, sendo que, a meta final a ser alcançada consiste em aplicá-los à um composto adicional, cujos detalhes estruturais não sejam bem conhecidos experimentalmente, de modo a utilizá-lo como auxílio para estudos experimentais.

\section{Localização da Energia Potencial Mínima}

Vários métodos são aplicados em mecânica molecular, para encontrar o mínimo da função de energia potencial. Estes métodos podem ser divididos em grupos, de acordo com a técnica empregada para localizar o mínimo de energia potencial:

- Métodos de Primeira Ordem: calculam apenas as primeiras derivadas da função de energia potencial.

- Métodos de segunda ordem: calculam as primeiras e segundas derivadas da função de energia potencial. Matematicamente, 
possuem condições necessárias e suficientes para satisfazer as condições de mínimos globais de diversas variáveis. As primeiras e segundas derivadas da função de energia potencial podem ser determinadas numericamente ou analiticamente, ou ainda, as primeiras derivadas avaliadas analiticamente e as segundas derivadas obtidas numericamente.

Existem vários artigos onde são discutidas as vantagens e desvantagens de uma técnica em relação a outra ${ }^{27-29}$. Os algoritmos mais utilizados em mecânica molecular são os métodos de Newton-Raphson (método de segunda ordem) e o "Steepest Descent" (método de primeira ordem). Ocasionalmente o algoritmo Simplex ${ }^{30}$ tem sido aplicado.

O objetivo desses métodos consiste em encontrar a coordenada $x$, onde a energia ( $f(x)$ ) é um mínimo, partindo de uma coordenada inicial, $x_{0}$.

A energia potencial, $\mathrm{U}$, para uma molécula com $\mathrm{N}$ átomos e $3 \mathrm{~N}$ coordenadas, escrita segundo a equação 1 , é expandida em série de Taylor, considerando até termos quadráticos, em torno do ponto de equilíbrio das coordenadas internas $\left(\mathrm{r}_{0}, \theta_{0}, \phi_{0}, \delta_{0}\right)$ :

$U=U_{0}+\sum\left(\frac{\partial U(r)}{\partial r}\right) \Delta r+\frac{1}{2} \sum\left(\frac{\partial^{2} U(r)}{\partial r^{2}}\right)_{0} \Delta r^{2}$

onde os termos cruzados estão negligenciados.

Para a localização do mínimo da função de energia potencial o uso de coordenadas atômicas cartesianas é mais favorável do que coordenadas internas, uma vez que, uma molécula com topologia qualquer, pode apresentar vários vínculos redundantes entre si. Uma forma eficaz e bastante utilizada para eliminar este problema, consiste em transformar os elementos, $\Delta$ (equação 19), em coordenadas cartesianas, as quais por sua condição de ortogonalidade, eliminam as relações de redundância existentes nas coordenadas internas.

A expressão geral para a energia potencial, U, em coordenadas cartesianas independentes, assume a seguinte forma:

$$
\mathrm{U}=\mathrm{U}_{0}+\sum \mathrm{A}_{\mathrm{i}}^{\alpha} \Delta X_{\mathrm{i}}^{\alpha}+\frac{1}{2} \sum \sum \mathrm{C}_{\mathrm{ij}}^{\alpha \beta} \Delta \mathrm{X}_{\mathrm{i}}^{\alpha} \Delta \mathrm{X}_{\mathrm{j}}^{\beta}
$$

onde $\mathrm{X}_{\mathrm{i}}^{\alpha}, \mathrm{X}_{\mathrm{j}}^{\beta}$ é o conjunto de coordenadas cartesianas $(\mathrm{i}, \mathrm{j}=1$, $\ldots, \mathrm{N} ; \alpha, \beta=\mathrm{x}, \mathrm{y}, \mathrm{z}), \mathrm{A}_{\mathrm{i}}^{\alpha}$ são formados pelos coeficientes que multiplicam os $\Delta \mathrm{X}_{\mathrm{i}}^{\alpha}$, os elementos da matriz $\mathrm{C}_{\mathrm{ij}}^{\alpha \beta}$ são todos os fatores que são multiplicados pelos $\Delta \mathrm{X}_{\mathrm{i}}^{\alpha}, \Delta \mathrm{X}_{\mathrm{j}}^{\beta}$.

Aplicando a condição necessária de mínimo, na energia potencial (equação 20):

$$
\frac{\partial \mathrm{U}}{\partial \Delta \mathrm{X}_{\mathrm{i}}^{\alpha}}=0
$$

um conjunto de $3 \mathrm{~N}$ equações algébricas lineares para os elementos, $\mathrm{X}_{\mathrm{i}}^{\alpha}$, é obtido:

$$
-\mathrm{A}_{\mathrm{i}}^{\alpha}=\sum \sum \mathrm{C}_{\mathrm{ij}}^{\alpha \beta} \Delta \mathrm{X}_{\mathrm{j}}^{\beta}
$$

o qual, devido as aproximações realizadas, deve ser resolvido iterativamente aplicando os métodos padrões (Método de Newton-Raphson, por exemplo). Os valores de $\Delta \mathrm{X}_{\mathrm{i}}^{\alpha}$ obtidos são usados para calcular o novo conjunto de coordenadas:

$\mathrm{X}_{\mathrm{i}}^{\alpha}($ novas $)=\mathrm{X}_{\mathrm{i}}^{\alpha}($ antigas $)+\Delta \mathrm{X}_{\mathrm{i}}^{\alpha}$

Utilizando os valores de $\mathrm{X}_{\mathrm{i}}^{\alpha}$ (novas), o processo (equação 19 - 23) é repetido, até que os valores, $\Delta X_{i}^{\alpha}$, da equação 23 sejam considerados suficientemente pequenos (próximos de zero). Na iteração final os valores $\mathrm{A}_{\mathrm{i}}^{\alpha}$ devem ser zero (ou muito próximo), de modo que as primeiras derivadas sejam nulas e somente permaneçam os termos $C_{i j}^{\alpha \beta}$.

A localização do mínimo de energia potencial requer somente $3 \mathrm{~N}-6$ graus de liberdade para a molécula, uma vez que as três translações e as três rotações não causam modificações na energia do sistema. Estes seis movimentos devem ser separados das vibrações internas, retirando-os da matriz das segundas derivadas. Para tratar a separação destes movimentos, existem, pelo menos, três métodos: "Método da Matriz Inversa Generalizada"26 "Método da Matriz Reduzida"17 e o Método desenvolvido por Thomas e Emerson ${ }^{31}$, utilizando as condições de Eckart ${ }^{32}$.

Os detalhes aritméticos da transformação da função de energia potencial de coordenadas internas para coordenadas cartesianas, bem como o comentário sobre os métodos de separação dos movimentos de rotação e translação das vibrações internas, estão descritos no trabalho de Machuca-Herrera ${ }^{33}$.

A energia potencial final em coordenadas cartesianas:

$$
\mathrm{U}=\mathrm{U}_{0}+\frac{1}{2} \sum \sum \mathrm{C}_{\mathrm{ij}}^{\alpha \beta} \Delta \mathrm{X}_{\mathrm{i}}^{\alpha} \Delta \mathrm{X}_{\mathrm{j}}^{\beta}
$$

pode então ser utilizada para calcular os números de onda vibracionais. Os números de onda em conjunto com os momentos de inércia, calculados a partir da geometria final, podem ser usados para calcular as contribuições rotacionais, translacionais e vibracionais, as quais são utilizadas na avaliação das funções termodinâmicas para o estado gasoso. A Figura 2 apresenta um fluxograma geral dos programas computacionais empregado nos cálculos de mecânica molecular.

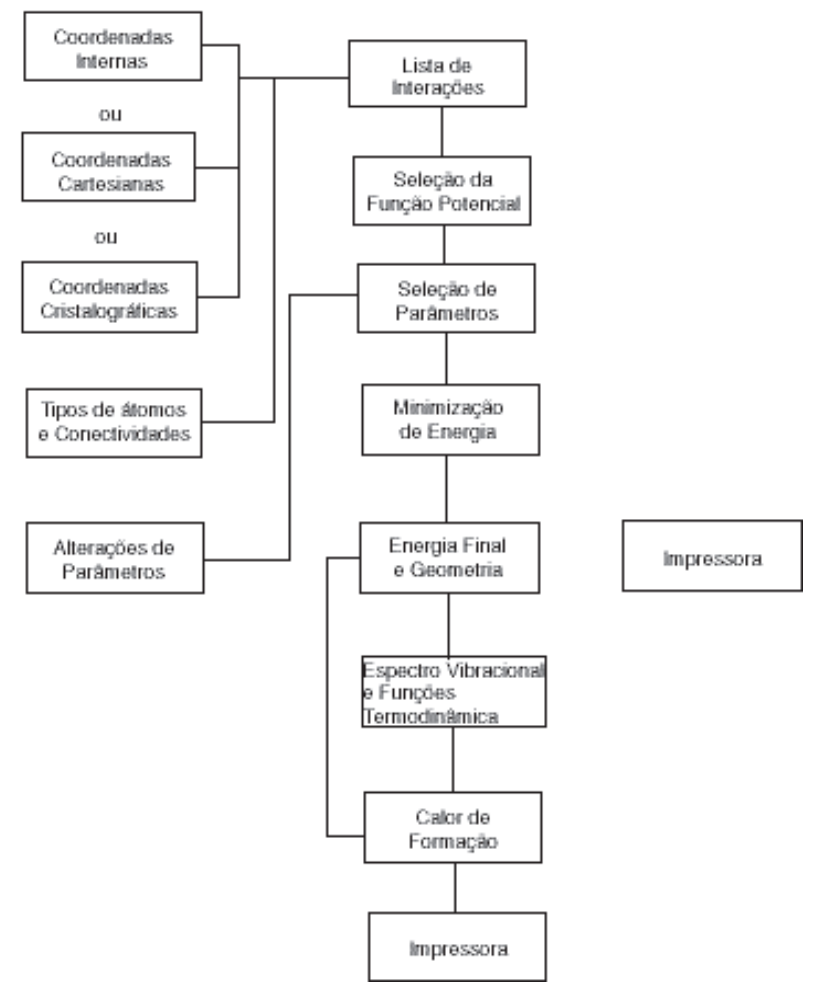

Figura 2. Fluxograma Geral dos Programas Computacionais de Mecânica Molecular.

\section{APLICAÇÕES DE MECÂNICA MOLECULAR EM QUÍMICẢ INORGÂNICA}

Cálculos de mecânica molecular são uma rotina em química orgânica e ultimamente vem sendo bastante empregado em química inorgânica ${ }^{34,35}$, visando estudos estruturais (previsão de estruturas desconhecidas), distribuição de isômeros e confôrmeros, 
seletividade de ligantes para íons metálicos, interações entre íons metálicos com proteínas e DNA. As aplicações de mecânica molecular aos sistemas inorgânicos tem sido apresentada em diversos artigos de revisão ${ }^{11,12,15,16,35,36}$.

A maioria dos compostos inorgânicos consiste de um ligante orgânico e um metal, desta forma os cálculos tem sido realizados utilizando um campo de força desenvolvido para moléculas orgânicas onde são adicionados os parâmetros necessários para a descrição das interações envolvendo o íon metálico, assumindo que o ligante não sofre modificações significativas quando coordenados ao metal. Estes parâmetros adicionais são obtidos por uma combinação de cálculos de mecânica quântica, estruturas cristalinas determinadas experimentalmente e espectros vibracionais (análise de coordenadas normais) ${ }^{37}$.

As preferências estruturais dos complexos são, em geral, função da estrutura do ligante. Cálculos de orbitais moleculares ao nível ab initio (Hartree-Fock) em modelos simples do tipo, M$\mathrm{O}(\mathrm{Me})_{2}$ e $\mathrm{M}-\mathrm{O}(\mathrm{Me})(\mathrm{Et})$, onde $\mathrm{M}=\mathrm{Li}, \mathrm{Na}, \mathrm{K}, \mathrm{Rb}, \mathrm{Cs}, \mathrm{Mg}, \mathrm{Ca}, \mathrm{Sr}$ e $\mathrm{Ba}^{38}$, revelaram uma preferência pela geometria trigonal planar, quando os oxigênios de éteres alifáticos são coordenados com cátions alcalinos e alcalinos terrosos. Essa preferência é forte em cátions pequenos e de alta valência e fraca em cátions grandes e de pequena valência. A partir dos resultados dos cálculos Hartree-Fock foi possível estabelecer uma extensão do MM3 para aplicação em complexos de éteres alifáticos com os cátions alcalinos $(\mathrm{Li}$ ao $\mathrm{Cs})$ e alcalinos terrosos $(\mathrm{Mg} \text { ao } \mathrm{Ba})^{38}$. O campo de força resultante reproduziu, entre outros fatores, as estruturas experimentais de 51 complexos diferentes de éteres polidentados com cátions alcalinos terrosos e alcalinos. A Tabela 1 compara as características estruturais calculadas e experimentais dos complexos de éter alifático com metais alcalinos e alcalinos terrosos. A Figura 3 apresenta dois éteres macrocíclicos analisados no trabalho de Hay e Rustad ${ }^{38}$.
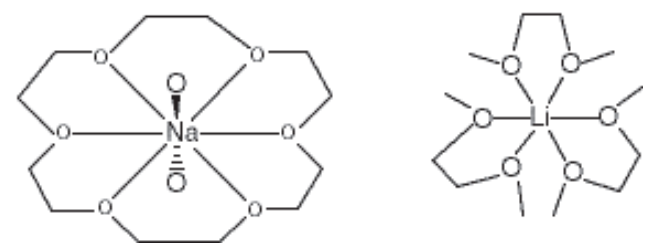

Figura 3. Éteres macrocíclicos ou éteres de "coroa".
Cálculos de mecânica molecular aplicados em compostos inorgânicos: organometálicos, compostos de coordenação e compostos bioinorgânicos são recentes, estando os campos de força, ainda em pleno desenvolvimento. Um amplo artigo de revisão ${ }^{39}$ expõe os trabalhos que envolvem cálculos de mecânica molecular aplicados à Química Bioinorgânica. Os responsáveis pela primeira publicação nessa área foram Buckinghan et alli ${ }^{40}$, na qual apresentaram os estudos da geometria molecular e estabilidades relativas de complexos que apresentam como ligante o trietileno tetramina-(S)-prolinato. Para a modelagem de centros metálicos em proteínas, Vedani et alli ${ }^{41}$ descreveram uma função potencial baseada em estudos de estruturas cristalinas de pequenas moléculas contendo quatro, cinco ou seis átomos de zinco ${ }^{42}$. A função inclui como variáveis as separações metal-ligante e os ângulos envolvendo o metal (no caso de distorções e transições entre geometrias de coordenação). Esta função foi incorporada ao programa de mecânica molecular YETI $^{43}$ e, foram feitos estudos de vários complexos de anidrase carbônica humana complexada e isolada. No trabalho de Vedani e Huhta44 foram descritos os resultados de uma análise do envolvimento de $\mathrm{Zn}(\mathrm{II})$ e $\mathrm{Co}(\mathrm{II})$ em estruturas cristalinas de pequenas moléculas, distinta da apresentada por Vedani et alli $^{41}$. Esses resultados formaram a base para uma função potencial estendida, visando modelar centros metálicos em proteínas. A nova função potencial foi também incorporada ao YETI (versão 4.5) e usada para modelar anidrase carbônica humana com $\mathrm{Co}(\mathrm{II})$. A Tabela 2 apresenta os parâmetros do Campo de Força para interações não-ligadas, utilizados no programa YETI (versão 4.5).

A aplicação de mecânica molecular em compostos inorgânicos apresenta limitações em função da necessidade de campos de força específicos e da dificuldade na definição de parâmetros. A derivação das constantes de força, a partir dos dados obtidos no espectro infravermelho é dificultada, isso porque a análise das baixas freqüências vibracionais da ligação metal ligante são freqüentemente complicadas por fortes efeitos eletrônicos e por sobreposição das vibrações do ligante ${ }^{45}$.

A utilização de métodos de Orbitais Moleculares ao nível $a b$ initio não tem sido muito divulgada devido a dificuldade na construção das bases para os centros metálicos. Cálculos aplicando o método do Funcional de Densidade vêm ganhando dimensão na determinação de parâmetros metálicos por apresentar melhor relação custo-benefício em relação a métodos $\boldsymbol{a b}$ initio $^{\mathbf{4 6}}$.

Tabela $1^{\text {a }}$. Características estruturais de anéis quelatos $\mathrm{O}-\mathrm{CH}_{2}-\mathrm{CH}_{2}-\mathrm{O}$ com metais alcalinos e alcalinos terrosos ${ }^{38}$.

\begin{tabular}{|c|c|c|c|c|c|c|c|c|}
\hline cátion & & $\mathrm{M}-\mathrm{O}^{\mathrm{b}}$ & $\mathrm{O}-\mathrm{C}^{\mathrm{b}}$ & $C-C^{b}$ & $\mathrm{O}-\mathrm{O}^{\mathrm{b}}$ & $\mathrm{O}-\mathrm{M}-\mathrm{O}{ }^{\mathrm{c}}$ & $\mathrm{M}-\mathrm{O}-\mathrm{C}^{\mathrm{c}}$ & $\mathrm{O}-\mathrm{C}-\mathrm{C}-\mathrm{O}^{\mathrm{c}}$ \\
\hline \multirow[t]{2}{*}{$\mathrm{Li}$} & exp. & 2,26 & 1,43 & 1,50 & 2,72 & 74 & 111 & 57 \\
\hline & calc. & - & 1,43 & 1,50 & 2,68 & 73 & 114 & 54 \\
\hline \multirow[t]{2}{*}{$\mathrm{Na}$} & exp. & 2,52 & 1,42 & 1,49 & 2,75 & 66 & 112 & 61 \\
\hline & calc. & - & 1,43 & 1,50 & 2,76 & 66 & 115 & 58 \\
\hline \multirow[t]{2}{*}{$\mathrm{K}$} & exp. & 2,83 & 1,42 & 1,48 & 2,81 & 59 & 114 & 65 \\
\hline & calc. & - & 1,43 & 1,50 & 2,82 & 60 & 116 & 62 \\
\hline \multirow[t]{2}{*}{$\mathrm{Rb}$} & exp. & 2,95 & 1,42 & 1,48 & 2,85 & 59 & 113 & 66 \\
\hline & calc. & - & 1,43 & 1,50 & 2,84 & 58 & 116 & 63 \\
\hline \multirow[t]{2}{*}{ Cs } & exp. & 3,19 & 1,42 & 1,48 & 2,87 & 54 & 113 & 68 \\
\hline & calc. & - & 1,43 & 1,50 & 2,86 & 53 & 118 & 65 \\
\hline \multirow[t]{2}{*}{$\mathrm{Mg}$} & exp. & 2,22 & 1,43 & 1,50 & 2,61 & 72 & 115 & 54 \\
\hline & calc. & - & 1,43 & 1,50 & 2,63 & 73 & 115 & 51 \\
\hline \multirow[t]{2}{*}{$\mathrm{Ca}$} & exp. & 2,51 & 1,44 & 1,49 & 2,69 & 65 & 116 & 56 \\
\hline & calc. & - & 1,43 & 1,50 & 2,72 & 65 & 117 & 54 \\
\hline \multirow[t]{2}{*}{$\mathrm{Sr}$} & exp. & 2,68 & 1,43 & 1,48 & 2,75 & 62 & 116 & 57 \\
\hline & calc. & - & 1,43 & 1,50 & 2,75 & 62 & 117 & 57 \\
\hline \multirow{2}{*}{$\underset{\mathrm{o}}{\mathrm{Ba}}$} & exp. & 2,87 & 1,43 & 1,49 & 2,78 & 58 & 116 & 60 \\
\hline & calc. & - & 1,43 & 1,50 & 2,79 & 58 & 118 & 59 \\
\hline
\end{tabular}

${ }^{\mathrm{a} O s}$ valores calculados são obtidos do programa MM3 modificado ${ }^{38}$;

${ }^{\mathrm{b}}$ Os valores de comprimentos de ligação são dados em ângstrons;

${ }^{\mathrm{c} O s}$ valores de ângulos são dados em graus. 
Tabela 2 (A, B e C). Parâmetros utilizados no programa YETI versão 4.5 , para interações não-ligadas ${ }^{44}$.

\begin{tabular}{cccc}
\hline A - PARÂMETROS DA LIGAÇÃO DE HIDROGÊNIO. \\
\hline tipo de ligação & distância de equilíbrio & $\mathrm{r}_{\mathrm{O}}{ }^{\mathrm{a}}$ & $\mathrm{E}_{0}{ }^{\mathrm{b}}$ \\
\hline O-H...O & 1,79 & 1,746 & $-4,946$ \\
O-H...N & 1,89 & 1,878 & $-4,655$ \\
O-H...S & 2,54 & 2,535 & $-1,733$ \\
N-H...O & 1,87 & 1,877 & $-4,073$ \\
N-H...N & 1,99 & 2,003 & $-3,491$ \\
N-H...S & 2,64 & 2,667 & $-1,455$ \\
S-H...O & 2,09 & 2,099 & $-2,328$ \\
S-H...N & 2,19 & 2,088 & $-2,037$ \\
S-H...S & 2,84 & 3,009 & $-1,164$ \\
\hline
\end{tabular}

${ }^{\mathrm{a}}$ Os raios estão calculados em ângstrons;

${ }^{\mathrm{b}}$ Parâmetro de Van der Waals $(\varepsilon)$ e Energia $\left(\mathrm{E}_{0}\right)$ em Kcal/mol.

\section{B. PARÂMETROS DE VAN DER WAALS.}

\begin{tabular}{ccc}
\hline tipo de átomo & raio de $\mathrm{vdW}^{\mathrm{b}}$ & $\varepsilon^{\mathrm{a}}$ \\
\hline H POLAR (H-O,H-N,H-S) & 1,00 & $-0,020$ \\
APOLAR (H-C) & 1,375 & $-0,038$ \\
\hline C > CH-, alifático & 1,85 & $-0,090$ \\
- $\mathrm{CH}_{2}$, alifático & 1,925 & $-0,120$ \\
- $\mathrm{CH}_{3}$, alifático & 2,00 & $-0,150$ \\
=CH-, aromático & 1,85 & $-0,120$ \\
$\mathrm{sp}^{2}>\mathrm{C}=$ aromático/alifático & 1,85 & $-0,120$ \\
sp $^{3}>$ C $>$ alifático & 1,80 & $-0,060$ \\
\hline $\mathrm{N}$ (CARGA FORMAL POSITIVA) & 1,85 & $-0,080$ \\
(CARGA FORMAL NEUTRA) & 1,75 & $-0,160$ \\
\hline O (CARBONILA) & 1,60 & $-0,120$ \\
NITRATO,FOSFATO,CARBOXILA & 1,60 & $-0,120$ \\
ÁGUA & 1,768 & $-0,152$ \\
ÉSTER,ÉTER,AÇÚCAR,HIDROXILA & 1,65 & $-0,150$ \\
\hline p & 2,10 & $-0,200$ \\
S (FORMA NEUTRA) & 2,00 & $-0,200$ \\
(ANIÔNICA (R-S $)$ ) & 2,00 & $-0,250$ \\
\hline Co(II) & 0,69 & $-0,014$ \\
\hline
\end{tabular}

${ }^{\mathrm{a} O s}$ raios estão calculados em ângstrons;

${ }^{\mathrm{b}}$ Parâmetro de Van der Waals $(\varepsilon)$ e Energia $\left(\mathrm{E}_{0}\right)$ em $\mathrm{Kcal} / \mathrm{mol}$.

\section{PARÂMETROS DOS CENTROS METÁliCOS.}

\begin{tabular}{ccccc}
\hline metal & ligante & NC & distância de equilíbrio & $\mathrm{E}_{0}{ }^{\mathrm{b}}$ \\
\hline $\mathrm{Zn}(\mathrm{II})$ & $\mathrm{O}$ & 4 & 1,961 & $-31,329$ \\
$\mathrm{Zn}(\mathrm{II})$ & $\mathrm{N}$ & 4 & 2,041 & $-35,143$ \\
$\mathrm{Zn}(\mathrm{II})$ & $\mathrm{S}$ & 4 & 2,317 & $-24,357$ \\
$\mathrm{Zn}(\mathrm{II})$ & $\mathrm{O}$ & 5 & 2,068 & $-22,262$ \\
$\mathrm{Zn}(\mathrm{II})$ & $\mathrm{N}$ & 5 & 2,126 & $-28,597$ \\
$\mathrm{Zn}(\mathrm{II})$ & $\mathrm{S}$ & 5 & 2,412 & $-17,792$ \\
$\mathrm{Co}(\mathrm{II})$ & $\mathrm{O}$ & 4 & 1,918 & $-37,368$ \\
$\mathrm{Co}(\mathrm{II})$ & $\mathrm{N}$ & 4 & 1,964 & $-40,838$ \\
$\mathrm{Co}(\mathrm{II})$ & $\mathrm{S}$ & 4 & 2,282 & $-29,096$ \\
\hline
\end{tabular}

${ }^{\mathrm{a}}$ Os raios estão calculados em ângstrons; ${ }^{\mathrm{b}}$ Parâmetro de Van der Waals $(\varepsilon)$ e Energia $\left(\mathrm{E}_{0}\right)$ em $\mathrm{Kcal} / \mathrm{mol}$.

Como a maioria dos compostos inorgânicos envolve interação entre ligante orgânico e metal, o procedimento usual em mecânica molecular para compostos deste tipo, consiste em utilizar os campos de força descritos para os compostos orgânicos, idênticos ou similares aos ligantes, adicionando à função potencial as considerações para a ligação metal ligante e os parâmetros que descrevem o íon metálico como a carga , a multiplicidade e, se presentes, o efeito trans e o efeito Jahn-Teller necessitam ser considerados. Comba \& Zimmer ${ }^{47}$ descreveram uma função potencial visando modelar efeitos Jahn-Teller em compostos $\mathrm{Cu}-\mathrm{N}_{6}$.

No trabalho de Comba e Bernhardt ${ }^{48}$ foi apresentado um campo de força apropriado para modelagem de complexos de $\mathrm{Cu}(\mathrm{II}), \mathrm{Ni}(\mathrm{II}), \mathrm{Co}(\mathrm{III}), \mathrm{Fe}(\mathrm{III}), \mathrm{Cr}(\mathrm{III}), \mathrm{Zn}$ (II) e $\mathrm{Rh}(\mathrm{III})$ com amina, carboxilato, piridina e tioéteres. Este campo de força apresenta as seguintes contribuições: deformação do ângulo da

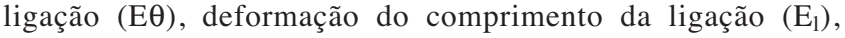
interações não-ligadas $\left(\mathrm{E}_{\mathrm{nl}}\right)$, deformação do ângulo de torção $\left(\mathrm{E}_{\omega}\right)$ e deformação fora do plano $\left(\mathrm{E}_{\mathrm{dfp}}\right)$, não sendo incluídas as interações eletrostáticas e ligações hidrogênio. A maioria dos parâmetros foi retirada dos trabalhos de Allinger ${ }^{6,7}$ e Hambley et alli $^{49}$ ou desenvolvidos por Comba e Bernhardt ${ }^{48}$ e incorporados ao programa MOMEC $87^{50}$. A Tabela 3 compara os comprimentos de ligação metal-Nitrogênio calculado pelo MOMEC 87 com os valores observados experimentalmente.

Tabela 3. Comprimentos da ligação (ângstrons) M(metal) - N (nitrogênio) em complexos de hexaaminas ${ }^{48}$.

\begin{tabular}{cccccc}
\hline metal & & diammac $^{\mathrm{a}}$ & $(\text { en })_{3}{ }^{\mathrm{b}}$ & $(\text { dien })_{2}{ }^{\mathrm{c}}$ & $\left(\mathrm{NH}_{3}\right)_{6}$ \\
\hline $\mathrm{Cr}(\mathrm{III})$ & calc. & 2,$05 ; 2,06$ & 2,08 & 2,$06 ; 2,08 ; 2,09$ & 2,08 \\
& obs. & 2,$04 ; 2,07$ & 2,07 & 2,$06 ; 2,08 ; 2,09$ & 2,08 \\
$\mathrm{Fe}(\mathrm{III})$ & calc. & 1,$97 ; 1,98$ & - & - & - \\
& obs. & 1,$97 ; 1,98$ & - & - & - \\
$\mathrm{NI}(\mathrm{II})$ & calc. & 2,$07 ; 2,11$ & 2,13 & 2,$10 ; 2,14 ; 2,15$ & - \\
& obs. & 2,$07 ; 2,12$ & 2,13 & 2,$07 ; 2,13 ; 2,18$ & - \\
$\mathrm{Zn}(\mathrm{II})$ & calc. & 2,$11 ; 2,20$ & 2,23 & - & - \\
& obs. & 2,$10 ; 2,21$ & 2,22 & - & - \\
$\mathrm{Rh}(\mathrm{II})$ & calc. & 2,$05 ; 2,06$ & 2,07 & - & 2,07 \\
& obs. & 2,$05 ; 2,06$ & 2,06 & - & 2,07 \\
\hline
\end{tabular}

${ }^{\text {adiammac }}=6,13$-dimetil-1,4,8,11-tetraazaciclotetradecano6,13-diamina;

$\mathrm{b}(\mathrm{en})_{3}=$ trisetilenodiamina;

${ }^{\mathrm{c}}(\text { dien })_{2}=$ bisetilenodiamina

Os cálculos de mecânica molecular podem também fornecer informações sobre os aspectos estereoespaciais em compostos de coordenação. Dados experimentais mostram que o budotitaniato $\left[\mathrm{Ti}(\mathrm{bzac})_{2}(\mathrm{OEt})_{2}\right]$ é uma droga promissora anticancerígena, no qual testes clínicos estão sendo efetuados em quimioterapia. Entretanto, sua estrutura é ainda desconhecida, apresentando 5 possíveis isômeros (Figura 4). No trabalho de Comba et alli ${ }^{51}$ foi elaborado um estudo da estabilidade relativa dos isômeros através do uso de mecânica molecular, permitindo uma análise quantitativa da distribuição dos isômeros e, conseqüentemente a determinação da estrutura molecular mais estável.
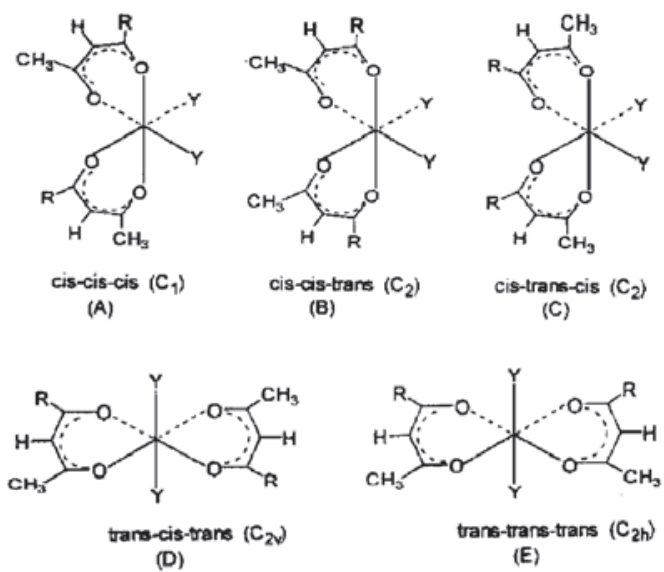

Figura 4. Estrutura dos isômeros do budotitaniato $\left[\text { Ti( }(\text { bzac })_{2}(\mathrm{OEt})_{2}\right]^{51}$. 
Em 1969, Bradley e Holloway52 analisaram, através de estudos espectroscópicos na região do infravermelho e estudos de RMN, aspectos da estabilização e labilidade de compostos do tipo $\left[\mathrm{M}(\mathrm{acac})_{2} \mathrm{Y}_{2}\right](\mathrm{acac}=\mathrm{RR}$ 'ac $=1,3$ dicetonas disubstituídas onde $\mathrm{R}=\mathrm{R}$ '= metila e $\mathrm{M}=\mathrm{Ti}, \mathrm{Ge}, \mathrm{Sn}$; $\mathrm{Y}=\mathrm{Cl}, \mathrm{Br}, \mathrm{I}$ ) verificando a maior estabilidade dos complexos de configuração cis , concordando com os resultados experimentais. Segundo Bradley \& Holloway ${ }^{52}$ embora fatores espaciais joguem a favor da configuração trans, resultados espectroscópicos de RMN mostram que compostos do tipo [Ti $\left.(\mathrm{acac})_{2}(\mathrm{X})_{2}\right](\mathrm{X}=\mathrm{OR}, \mathrm{Cl}, \mathrm{Br}$, I e R $=\mathrm{Me}, \mathrm{Et}, \mathrm{Pr}$ ), são encontradas preferencialmente na configuração cis, portanto efeitos espaciais desfavoráveis não influem na estabilização da configuração cis. David et alli ${ }^{53}$ determinaram por cristalografia de raios-X que a ligação $\mathrm{Ti}$ - $\mathrm{O}$ no acetilacetonato é ligeiramente assimétrica , sugerindo alguma perda de deslocalização de elétrons (Figura 5). A doação $\pi$ do acetilacetonato e grupos substituintes análogos, ao metal central, foi proposta como explicação da seletividade da geometria cis no Ti $\left[(R R \text { 'ac })_{2} \mathrm{X}_{2}\right]^{\mathrm{n}+}$. O estudo dos $\beta$ dicetonatos de Ti (IV) é dificultado pois nenhum composto de Ti(IV), nem ligantes do tipo acetilacetonato, foram parametrizados para cálculos de mecânica molecular. Desta forma, foi necessário estabelecer um conjunto de parâmetros para os complexos $\beta$ dicetonatos de $\mathrm{Co}(\mathrm{III})$, para os quais um campo de força estava estabelecido ${ }^{48}$, para posteriormente descrever um campo de força correspondente ao Ti(IV $)^{51}$. Para os parâmetros $\mathrm{Co}-\mathrm{N}$, foi utilizada várias aminascarboxiladas, existindo uma excelente concordância entre a geometria calculada e a experimental. A influência trans exercida pelos grupos oxo e alcóxido, devem ser incluída nos cálculos, e foi determinada através da definição de diferentes tipos de átomos de oxigênio no acetilacetonato cis ou trans com ligantes trans diretores, utilizando o programa de modelagem molecular MOMEC $^{54}$. Os resultados estão presentes na Tabela $4^{51}$.

A distribuição de isômeros determinada através de cálculos de mecânica molecular é feita a partir das estabilidades relativas dos diferentes isômeros analisados ${ }^{51}$. A confiabilidade dos resultados é baseada na elaboração de um campo de força com parâmetros bem definidos. A construção do campo de força para os complexos $\beta$ dicetonatos de $\operatorname{Co}($ III) foi facilitada, em função da existência dos estudos, envolvendo cálculos de mecânica molecular, para complexos de Co(III), principalmente com ligantes aminas ${ }^{48}$. Desta forma a distribuição de isômeros para os $b$ dicetonatos pode ser prevista. Cabe ressaltar que, os efeitos ambientais (p. ex.: solvatação) não foram incluídos, os cálculos são realizados supondo os sistemas em fase gasosa.

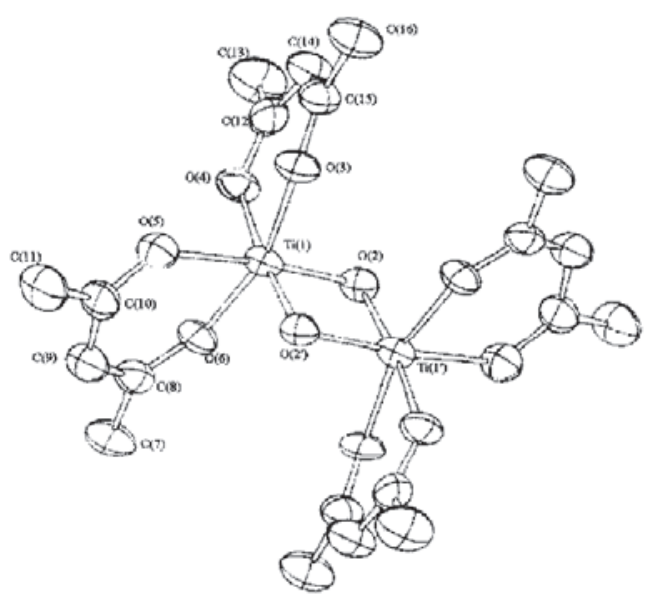

Figura 5. Estrutura do $\left[\mathrm{Ti}(\text { acac })_{2} \mathrm{O}\right]_{2}$, onde acac $=2,4$ pentadionato ${ }^{51}$.

Na Tabela 5 pode ser observada a ótima concordância entre os resultados obtidos com o uso de mecânica molecular, na previsão de isômeros de complexos de Ti(IV), e os dados experimentais correspondentes. A comparação relativa da energia potencial total dos isômeros com ligantes $\beta$ dicetonatos, dispostos na posição cis e trans, apresenta grande importância, uma vez que, esta energia

Tabela 4. Parâmetros estruturais calculados e observados dos complexos b-dicetonatos de $\operatorname{Ti}(\mathrm{IV})^{\mathrm{a}}$ e Co(III) ${ }^{\mathrm{a}}$

\begin{tabular}{|c|c|c|c|}
\hline composto & parâmetro & observado ${ }^{b}$ & calculado $^{\mathrm{b}}$ \\
\hline \multirow[t]{2}{*}[\mathrm{Ti}(\mathrm{acac})_{3}]{$^{+}$} & Ti4-ODC1 & 1,94 & 1,97 \\
\hline & ODC1-Ti4-ODC1 & 85 & 92 \\
\hline \multirow{3}{*}[\mathrm{Ti}(\mathrm{acac})_{2}\mathrm{O}]{$_{2}$} & Ti-ODC1 & 1,$98 ; 1,97$ & 1,98 \\
\hline & Ti4-ODC2 & 2,$04 ; 2,06$ & 2,04 \\
\hline & $\mathrm{O}^{\text {oxo }}-\mathrm{Ti} 4-\mathrm{O}^{\text {oxo }}$ & $82 ; 83$ & 89 \\
\hline \multirow{5}{*}[\mathrm{Ti}(\mathrm{bzac})_{2}(\mathrm{OEt})_{2}]{$^{\mathrm{c}}$} & Ti4-ODC1 & & 1,98 \\
\hline & Ti4-ODC2 & & 2,04 \\
\hline & Ti4-OS & & 1,80 \\
\hline & ODC1-Ti4-ODC2 & & 89 \\
\hline & OS-Ti4-OS & & 95 \\
\hline \multirow{3}{*}[\mathrm{Ti}(\mathrm{bbac})_{2}(\mathrm{OEt})_{2}]{} & Ti4-ODC1 & 1,99 & 1,98 \\
\hline & Ti4-ODC2 & 2,06 & 2,04 \\
\hline & ODC1-Ti4-ODC2 & 82 & 87 \\
\hline \multirow{2}{*}[\mathrm{Co}(\mathrm{acac})_{3}]{$^{+\mathrm{c}}$} & Co3-ODC1 & 1,89 & 1,89 \\
\hline & ODC1-Co3-ODC1 & 97 & 95 \\
\hline \multirow[t]{3}{*}[\mathrm{Co}(\mathrm{acac})_{2}(\mathrm{en})]{$^{+\mathrm{c}}$} & Co3-ODC1 & 1,89 & 1,89 \\
\hline & Co3-N & 1,94 & 1,95 \\
\hline & OCD1-Co3-OCD1 & 96 & 94 \\
\hline \multirow{3}{*}[\mathrm{Co}(\mathrm{acac})_{2}(\mathrm{NH}_{3})_{2}]{$^{+\mathrm{c}}$} & Co3-ODC1 & 1,90 & 1,89 \\
\hline & Co3-N & 1,96 & 1,95 \\
\hline & ODC1-Co3-ODC1 & 96 & 94 \\
\hline \multirow{4}{*}[\mathrm{Co}(\mathrm{bzac})_{2}(\mathrm{en})]{$^{+\mathrm{c}}$} & Co3-ODC1 & & 1,89 \\
\hline & $\mathrm{Co} 3-\mathrm{N}$ & & 1,95 \\
\hline & ODC1-Co3-ODC1 & & 94 \\
\hline & ODC1-Co3-N & & $175 ; 90$ \\
\hline
\end{tabular}

a) para nomenclatura ver Figura 6;

b) média dos ângulos em graus e distancia em Angstron,;

c) ref. 51 


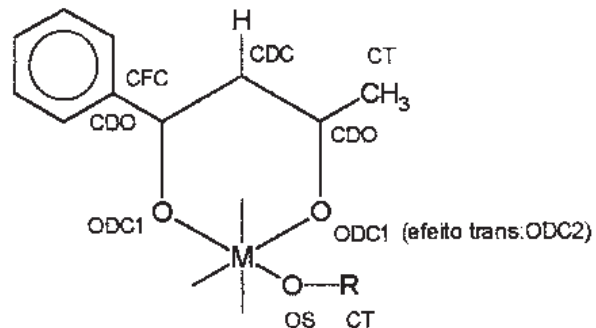

Figura 6. Geometria dos complexos b-dicetonatos de Ti(IV) e Co(III) (Para parâmetros de distâncias de ligação ver tabela 4).

Tabela 5. Distribuição de isômeros dos complexos b dicetonatos de $\mathrm{Co}(\mathrm{III})$ e $\mathrm{Ti}(\mathrm{IV})$.

\begin{tabular}{cccc}
\hline composto & isômero & $\%$ calculada & $\%$ observada \\
\hline$\left[\mathrm{Co}(\mathrm{acac})_{2}\left(\mathrm{NH}_{3}\right)_{2}\right]^{+}$ & cis & 69 & 73 \\
& trans & 31 & 27 \\
{$\left[\mathrm{Ti}(\mathrm{bzac})_{2}(\mathrm{OEt})_{2}\right]$} & $\mathrm{A}$ & 57 & 60 \\
& $\mathrm{~B}$ & 17 & 19 \\
& $\mathrm{C}$ & 26 & 21 \\
& $\mathrm{D}$ & 0 & 0 \\
{$\left[\mathrm{Ti}(\mathrm{bbac})_{2}(\mathrm{OEt})_{2}\right]$} & $\mathrm{E}$ & 0 & 0 \\
& cis & 100 & 100 \\
& trans & 0 & 0 \\
\hline
\end{tabular}

pode ser relacionada com as estabilidades relativas dos compostos de coordenação.

De acordo com a distribuição de isômeros a resolução da geometria do budotitaniato é apresentada na Figura 7.

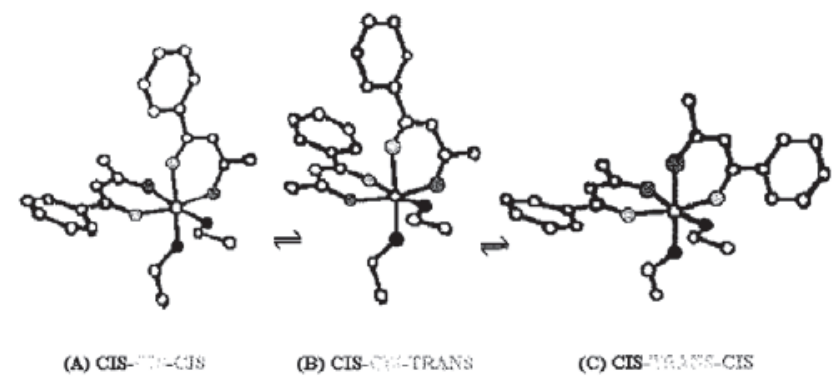

Figura 7. Geometria dos três isômeros do budotitaniato mais estáveis.

A metodologia de mecânica molecular também tem sido usada no estudo de modelos de compostos de coordenação que transportam ferro em sistemas vivos ${ }^{55}$ e no estudo de estabilidade de metaloporfirinas ${ }^{56}$, mostrando a diversidade de sistemas que podem ser avaliados por esta metodologia de cálculo.

\section{CONCLUSÕES}

Apesar das limitações existentes, como a dificuldade de parametrização, os métodos do Campo de Força Empírico têm sido frequientemente escolhidos para o estudo de sistemas complexos tais como compostos de coordenação e organometálicos. Isso deve-se aos fatos dos métodos quânticos, seja a nível ab initio ou semi-empírico, apresentam elevado custo associado ao tempo computacional

Ao longo de duas décadas, o desenvolvimento e aprimoramento de técnicas de mecânica molecular vêm se tornando poderosa ferramenta computacional aplicada à Química Inorgânica para prever estruturas e suas respectivas energias. Logo, é necessário avaliar o que se deseja obter com os cálculos, antes de escolher o método computacional.

É importante ressaltar a relevância da comparação dos resultados obtidos da mecânica molecular com dados experimentais ou mesmo com resultados de métodos quânticos, para obtenção de parâmetros de avaliação da eficiência do mesmo. Nos trabalhos com mecânica molecular, em geral, é escolhido um composto representativo de sua classe, cujos parâmetros calculados possam ser comparados com valores experimentais. A partir daí, os estudos deste composto modelo podem ser estendidos a toda a classe de origem.

Enfim, a mecânica molecular vêm se tornando cada vez mais eficaz com a ajuda de programas mais refinados e computadores mais potentes, e vêm cumprindo seu papel na determinação de geometrias moleculares mais estáveis, com baixo custo computacional.

\section{AGRADECIMENTOS}

Os autores agradecem à FAPERJ, Fundação Universitária José Bonifácio (FUJB) e a CAPES pelo suporte financeiro.

\section{REFERENNCIAS}

1. Bartell, L. S; Jacob, E. J.; Thompson, H. B.; J. Chem. Phys. 1967, 47, 3736.

2. Boyd, R. H.; J. Chem. Phys. 1968, 49, 2574.

3. Allinger, N. L.; Sprage, J. T.; J. Am. Chem. Soc. 1973, 95, 3893.

4. Schleyer, P. Von R.; Engler, E. M.; Andose, J. D.; J. Am. Chem. Soc. 1973, 95, 8005

5. Bartell, L. S.; Fitzwater, S.; J. Am. Chem. Soc. 1976, 98, 5107.

6. Allinger, N. L.; J. Am. Chem. Soc. 1977, 99, 8127.

7. Allinger, N. L.; Zhu, Z. S.; Chen, K.; J. Am. Chem. Soc. $1992,114,6120$.

8. Snow, M. R.; J. Am. Chem. Soc. 1970, 92, 3610.

9. Snow, M. R.; Buckingham, D. A.; Marzilli, P. A.; Sargeson, A. M.; J. Chem. Soc.; Chem. Commun. 1969, 891.

10. Buckingham, D. A.; Maxwell, I. E.; Sargeson, A. M.; Snow, M. R.; J. Am. Chem. Soc. 1970, 92, 3617.

11. Brubaker, G. R.; Johnson, D. W.; Coord. Chem. Rev. 1984, 53, 1 .

12. Hancock, R. D.; Prog. Inorg. Chem. 1989, 37, 187.

13. Hambley, T. W.; Comm. Inorg. Chem. 1992, 14, 1.

14. Comba, P.; Coord. Chem. Rev. 1993, 123, 1.

15. Hay, B. P.; Coord. Chem. Rev. 1993, 126, 177.

16. Comba, P.; Comm. Inorg. Chem. 1994, 16, 133.

17. Boyd, R. H.; Breitting, S. M.; Mansfield, M.; A. I. Ch. Eng. 1973, 19, 1016.

18. Boyd, D. B.; Lipkowitz, K. B.; J. Chem. Educ. 1982, $59,269$.

19. Rasmunssen, K.; Niketic, S. R.; The Consistent Force Field: A Documentation; Springer Verlag; Berlin 1977.

20. Ermer, O.; Structure and Bonding; Vol.27, Springer Verlag; Berlin 1976.

21. Burkert, U.; Allinger, N. L.; Molecular Mechanics; ACS Monograph No.177; Washington, D. C. 1982.

22. Osawa, E.; Musso, H.; Angew. Chem. 1983, 22, 1.

23. Clark, T.; A Handbook of Computational Chemistry; Wiley; New York 1985.

24. Rappé, A. K.; Casewit, C. J.; Colwell, K. S.; Godart III, W. A.; Skiff, W. M.; J. Am. Chem. Soc. 1992, 114, 10024.

25. Grant, G. H. ; Richards, W. H.; Computational Chemistry, Oxford University Press 1995.

26. Lifson, S.; Warshel, A.; J. Chem. Phys. 1968, 49, 5116.

27. Ermer, O.; Angew. Chem. 1977, 16, 798.

28. Ermer, O.; Lifson; S.; J. Am. Chem. Soc. 1973, 95, 4121.

29. White, D. N. J.; Bovill, M. J.; J. Chem. Soc. Perkin Trans. II 1977, 1610.

30. Brunel, Y.; Faucher, H.; Gagnaire, D.; Rassat, A.; Tetrahedron 1975, 31, 1075. 
31. Thomas M. W.; Emerson, D.; J. Mol. Struct 1973, 16, 473. 32. Echart, C.; Phys. Rev. 1935, 47, 552.

33. Machuca-Herrera, J. O.; Tese de Doutorado, Instituto de Química, Universidade Estadual de Campinas, Campinas, SP, 1988.

34. Brandt, P.; Norrby, T; Åkermark, B.; Inorg. Chem. 1998, $37,4120$.

35. Comba, P.; Hambley, T. W.; Molecular Modelling of Inorganic Compounds, VCH; Weinheim 1995.

36. Comba, P.; Coord. Chem. Rev. 1993, 123, 1.

37. Allured, V. S.; Kelly, C. M.; Landis, C. R.; J. Am. Chem. 1991, 113, 1 .

38. Hay, B. P.; Rustad, J. R. ; J. Am. Chem. Soc. 1994, 116, 14.

39. Zimmer, M.; Chem. Rev. 1995, 95, 8.

40. Buckingham, D. A.; Maxwell, I. E.; Sargeson, A. M.; Smith, M. R.; J. Am. Chem. Soc. 1970, 92, 3617.

41. Vedani, A.; Dobler, M. E.; Dunitz, J. D.; J. Comput. Chem. 1986, 7, 701.

42. Allen, F. H.; Bellard, A.; Brice, M. D.; Cartwright, B. A.; Dubleday, A.; Hummelink, T.; Peters, B. G; Bennard, O.; Motherwell, W. D. S.; Rodgers, J. R.; Watson, D. G.; Acta Crystallogr.; Sect. B 1979, 35, 2331.

43. Vedani, A.; Dunitz, J. D.; J. Am. Chem. Soc. 1985, 107, 7653.
44. Vedani, A.; Huhta, D. W.; J. Am. Chem. Soc. 1990, 112, 12. 45. Nakamoto, K.; Infrared and Raman Spectra of Inorganic and Coordination Compounds; 3rd ed: Wiley; New York 1978.

46. Custódio, R.; Morgon, N. H.; Quím. Nova 1995, 18, 44.

47. Comba, P.; Zimmer, M.; Inorg .Chem. 1994, 33, 5368.

48. Comba, P.; Bernhardt, P. V.; Inorg. Chem. 1992, 31, 2638.

49. Hambley, T. W.; Hawkins, C. J.; Palmer, J. A.; Snow, M. R.; Aust. J. Chem. 1981, 34, 4.

50. Hambley, T. W.; MOMEC87, A Program for Strain Energy Minimization; University of Sydney: Sydney, Austrália 1987.

51. Comba, P.; Jakob, H.; Keppler, B. K.; Nuber, B.; Inorg. Chem 1994, 33, 3396.

52. Bradley, D. C.; Holloway, C. E.; J. Chem. Soc. 1969, 282.

53. Smith, G. D.; Caughlan, C. N.; Campbell, J. A.; Inorg. Chem. 1972, 11, 2989.

54. Comba, P.; Hambley, T. W.; Okon, N. MOMEC, A Strain Energy Minimization Package Adapted to Hyperchem ${ }^{\mathrm{TM}}$, Altenhoff\&Schmitz, Dortmund Germany (1995).

55. Zinelabidine, A.; Bouraoui, A.; Mhenni, F.; Blaive, B.; Gallo, R.; J. Mol. Struct. (Theochem.) 1993, 286, 267.

56. Machado, S. de P.; Cardoso, J. N.; Machuca Herrera J. O.; Quím. Nova 1994, 17, 152. 